\title{
Campo escalar complejo masivo de Klein-Gordon en un background de Schwarzschild
}

\author{
Carlos A. Marín ${ }^{1 *}$ \\ ${ }^{1}$ Colegio de Ciencias e Ingeniería, Universidad San Francisco de Quito \\ *Autor principal/Corresponding author, correo electrónico: cmarin@usfq.edu.ec \\ Editado por/Edited by: Cesar Zambrano, Ph.D. \\ Recibido/Received: 2015/02/27. Aceptado/Accepted: 2015/03/05. \\ Publicado en línea/Published online: 2015/05/22. Impreso/Printed: 2015/06/01.
}

\begin{abstract}
The behaviour of a massive complex Klein-Gordon scalar field in a Schwarzschild background is analized using tortoise coordinates. Using these coordinates we obtain an expression for the action in terms of an effective potential and a radial wave function. From the action we get the corresponding wave equation and obtain some solutions of that equation for a mode of frequency $\omega$. Additionally, we study the effective potential in terms of the radial distance $r$ and the quantum number $l$. Finally, we estimate the energy that we need to traverse the potential barrier for some values of $l$.
\end{abstract}

Keywords. Complex scalar field, Klein-Gordon, general relativity, Schwarzschild.

\section{Massive complex Klein-Gordon scalar field in a Schwarzschild background}

\section{Resumen}

El comportamiento de un campo escalar complejo masivo de Klein-Gordon inmerso en un background de Schwarzschild es analizado utilizando coordenadas de tortuga. Usando dichas coordenadas obtenemos una expresión para la acción en términos de un potencial efectivo y una función de onda radial. A partir de dicha acción deducimos la ecuación de onda correspondiente y calculamos algunas soluciones de la mencionada ecuación para un modo de frecuencia $\omega$. Adicionalmente, se estudia el comportamiento del potencial efectivo en función de la distancia radial $r$ y el número cuántico $l$. Finalmente, evaluamos la energía necesaria para atravesar la barrera de potencial para algunos valores de $l$.

Palabras Clave. Campo escalar complejo, Klein-Gordon, relatividad general, Schwarzschild.

\section{Introducción}

Para la formulación de la Teoría General de la Relatividad (TGR), Einstein se basó en el principio de equivalencia entre la masa inercial y la masa gravitacional. Einstein se dio cuenta de que esta equivalencia sólo podía mantenerse si existía una conexión entre la fuerza gravitatoria y la geometría del espacio. La genealidad de Einstein fue suponer que el espacio-tiempo localmente no es plano, sino que está curvado debido a la distribución de materia y energía en él presente. En la TGR el espacio y el tiempo constituyen una estructura geométrica curvada en forma continua. La gravedad es una distorsión de la curvatura del espacio-tiempo.

La TGR se resume en 14 ecuaciones [1-6]. Las ecuaciones de campo de Einstein (que representan en total 10 ecuaciones escritas en forma tensorial)

$$
R_{\mu \nu}-\frac{1}{2} R g_{\mu \nu}-\lambda g_{\mu \nu}=\frac{8 \pi G}{c^{4}} T_{\mu \nu}
$$

y la ecuación de las geodésicas (cuatro ecuaciones)

$$
\frac{d^{2} x^{\mu}}{d s^{2}}+\Gamma_{\rho \sigma}^{\mu}\left(\frac{d x^{\rho}}{d s}\right)\left(\frac{d x^{\sigma}}{d s}\right)=0
$$

En (1) $R=g^{\alpha \beta} R_{\alpha \beta}$ es el escalar de Ricci. A $R_{\mu \nu}$ se le denomina Tensor de Ricci, el cual se define a partir del Tensor de Riemann-Christoffel $\left(R_{\nu \rho \sigma}^{\mu}\right)$ que determina la curvatura del espacio-tiempo: $R_{\mu \nu}=R_{\mu \alpha \nu}^{\alpha}$ donde

$$
\begin{aligned}
R_{\nu \rho \sigma}^{\mu}=\frac{\partial}{\partial x^{\rho}} & \left(\Gamma_{\nu \sigma}^{\mu}\right)-\frac{\partial}{\partial x^{\sigma}}\left(\Gamma_{\nu \rho}^{\mu}\right) \\
& +\Gamma_{\alpha \rho}^{\mu} \Gamma_{\nu \sigma}^{\alpha}-\Gamma_{\nu \rho}^{\alpha} \Gamma_{\alpha \sigma}^{\mu}
\end{aligned}
$$

$g_{\mu \nu}$ es el tensor métrico; este determina las propiedades métricas del espacio-tiempo. $T_{\mu \nu}$ es el tensor energíacantidad de movimiento. $G$ es la constante de gravitación universal, $c$ es la velocidad de la luz y $\lambda$ es la constante cosmológica introducida por Einstein en 1917. En la ecuación (2) $s$ representa el arco de curva y $\Gamma_{\rho \sigma}^{\mu}$ son 
los símbolos de Christoffel de segunda especie

$$
\Gamma_{\rho \sigma}^{\mu} \equiv \frac{1}{2} g^{\mu \alpha}\left(\frac{\partial g_{\rho \alpha}}{\partial x^{\sigma}}+\frac{\partial g_{\sigma \alpha}}{\partial x^{\rho}}-\frac{\partial g_{\rho \sigma}}{\partial x^{\alpha}}\right)
$$

$x^{\mu}$ es el cuadrivector posición de la partícula. Las letras griegas como: $\mu, \nu, \rho$, etc. toman los valores $0,1,2$ y $3 . \mathrm{Si}$ hacemos $\lambda=0$ en la ecuación (1), tenemos

$$
G_{\mu \nu} \equiv R_{\mu \nu}-\frac{1}{2} R g_{\mu \nu}=\frac{8 \pi G}{c^{4}} T_{\mu \nu}
$$

A $G_{\mu \nu}$ se le denomina Tensor de Einstein. Escrita en esta forma la última ecuación nos dice que la curvatura de una región del espacio-tiempo está determinada por la distribución de masa y energía que existe en la misma.A partir de las ecuaciones de campo, se puede determinar la métrica del espacio-tiempo para una distribución dada de materia o energía (el tensor métrico describe la desviación del teorema de Pitágoras en un espacio curvo). Para una distribución de masa con simetría esférica la métrica correspondiente es la de Schwarzschild.

En Mecánica Cuántica Relativista, la ecuación de KleinGordon [7-9]

$$
\left(\partial_{\mu} \partial^{\mu}+m^{2}\right) \phi=0
$$

donde $m^{\prime}=\frac{m_{0} c}{\hbar}$ describe un campo escalar real de espín cero con masa $m_{0}$ ( $\hbar$ es la constante de Planck reducida) en un espacio-tiempo plano. Dicha ecuación se puede derivar del Lagrangiano

$$
L=\frac{1}{2}\left(\partial_{\mu} \phi\right)\left(\partial^{\mu} \phi\right)-\frac{1}{2} m^{\prime 2} \phi^{2}
$$

En el caso de un campo escalar complejo y masivo en un espacio-tiempo plano el Lagrangiano es

$$
L=\left(\partial_{\mu} \chi\right)\left(\partial^{\mu} \chi^{*}\right)-m^{\prime 2} \chi^{*} \chi
$$

donde

$$
\begin{aligned}
\chi & =\frac{1}{\sqrt{2}}\left(\chi_{1}+i \chi_{2}\right) \\
\chi^{*} & =\frac{1}{\sqrt{2}}\left(\chi_{1}-i \chi_{2}\right)
\end{aligned}
$$

de manera que las ecuaciones de Euler-Lagrange conducen a las ecuaciones de Klein-Gordon

$$
\left(\partial_{\mu} \partial^{\mu}+m^{\prime 2}\right) \chi=0
$$

$\mathrm{y}$

$$
\left(\partial_{\mu} \partial^{\mu}+m^{\prime 2}\right) \chi^{*}=0
$$

En la referencia [10] se analiza de manera elemental el comportamiento de un campo escalar real de Klein Gordon desprovisto de masa en un background de Schwarzschild usando coordenadas de tortuga ( tortoise coordinates). En el presente artículo generalizamos dicho estudio para el caso de un campo escalar complejo masivo en dicho background. En primer lugar procederemos a calcular la expresión de la acción (usando coordenadas de tortuga) en términos del potencial efectivo, y a partir de la misma deduciremos la ecuación de onda correspondiente. Posteriormente analizaremos algunas soluciones de dicha ecuación y calcularemos los puntos críticos del potencial efectivo. Finalmente evaluaremos la energía que es necesaria para atravesar la barrera de potencial para algunos valores del número cuántico $l$ cerca del horizonte de sucesos dado por la fórmula de Schwarzschild $r_{s}=\frac{2 G M}{c^{2}}$.

\section{Métrica (Coordenadas de tortuga)}

Consideremos la métrica de Schwarzschild [2, 3]:

$$
\begin{array}{r}
(d s)^{2}=c^{2}(d \tau)^{2}=\gamma c^{2}(d t)^{2}-\gamma^{-1}(d r)^{2} \\
-r^{2}(d \theta)^{2}-r^{2} \sin ^{2} \theta(d \phi)^{2}
\end{array}
$$

donde $d s$ representa el intervalo invariante, $c$ es la velocidad de la luz, $r, \theta$ y $\varphi$ son las coordenadas esféricas de un punto en el espacio, $\tau$ es el tiempo propio (el intervalo de tiempo medido en un sistema de referencia donde el reloj está en reposo) y $t$ es el tiempo coordenado medido por un observador remoto en reposo en dicho punto. $\gamma=1-\frac{2 G M}{r c^{2}}$, donde $M$ representa la masa que genera el campo gravitacional y $G$ es la constante de gravitación universal. Al introducir la coordenada radial

$$
r^{*}=r+\frac{2 G M}{c^{2}} \ln \left(\frac{r-\frac{2 G M}{c^{2}}}{\frac{2 G M}{c^{2}}}\right),
$$

vemos que cuando nos aproximamos al horizonte de sucesos del agujero negro definido por el radio de Schwarzschild $r \rightarrow r_{s}=\frac{2 G M}{c^{2}}$, dicha coordenada radial se corre hacia $-\infty$. El sistema coordenado resultante $r^{*}, \theta, \varphi$ cubre entonces únicamente la region $r>\frac{2 G M}{c^{2}}$. A la coordenada radial $r^{*}$ se le denomina coordenada de tortuga (tortoise coordinate).

Diferenciando la ecuación (14) obtenemos $d r^{*}=\frac{d r}{\gamma} \mathrm{y}$ por lo tanto podemos escribir

$$
\frac{(d r)^{2}}{\gamma}=\gamma\left(d r^{*}\right)^{2}
$$

de manera que la métrica (13) se puede expresar como:

$$
\begin{array}{r}
(d s)^{2}=c^{2}(d \tau)^{2}=\gamma\left[c^{2}(d t)^{2}\right. \\
\left.-\left(d r^{*}\right)^{2}\right] \\
-r^{2}(d \Omega)^{2}
\end{array}
$$

donde $(d \Omega)^{2}=(d \theta)^{2}+\sin ^{2} \theta(d \varphi)^{2}$

La parte radial y temporal de la métrica (para valores fijos de $\theta$ y $\varphi$ ) tiene ahora una forma simple denominada "métrica conformalmente plana" [10]. 


\section{Acción}

Consideremos un campo escalar complejo masivo (libre) de Klein Gordon $(\chi)$ inmerso en un background de Schwarzschild. La acción para $\chi$ es

$$
S=\int d^{4} x \sqrt{-g}\left(g^{\mu \nu} \partial_{\mu} \chi^{*} \partial_{\nu} \chi-m^{\prime 2} \chi^{*} \chi\right)
$$

donde $m^{\prime}=\frac{m_{0} c}{\hbar}$ ( $m_{0}$ es la masa del campo escalar complejo y $\hbar$ es la constante de Planck reducida). Los índices $\mu, \nu$ pueden tomar los valores $0,1,2$ y $3 ; d^{4} x=$ $d x^{0} d x^{1} d x^{2} d x^{3}\left(x^{0}=c t, x^{1}=r, x^{2}=\theta\right.$ у $\left.x^{3}=\phi\right)$.

$\left(g^{\mu \nu}\right)=\left(\begin{array}{cccc}\gamma^{-1} & 0 & 0 & 0 \\ 0 & -\gamma & 0 & 0 \\ 0 & 0 & -r^{-2} & 0 \\ 0 & 0 & 0 & -\left(r^{2} \sin ^{2} \theta\right)^{-1}\end{array}\right)$

El tensor covariante correspondiente es

$\left(g_{\mu \nu}\right)=\left(\begin{array}{cccc}\gamma & 0 & 0 & 0 \\ 0 & -\gamma^{-1} & 0 & 0 \\ 0 & 0 & -r^{2} & 0 \\ 0 & 0 & 0 & -r^{2} \sin ^{2} \theta\end{array}\right)$

por otro lado,

$$
g=\operatorname{det}\left(g_{\mu \nu}\right)=-r^{4} \sin ^{2} \theta
$$

de manera que $\sqrt{-g}=r^{2} \sin \theta$. Usando la regla de la cadena tenemos $\frac{\partial \chi}{\partial r}=\left(\frac{\partial \chi}{\partial r^{*}}\right)\left(\frac{d r^{*}}{d r}\right)=\frac{1}{\gamma}\left(\frac{\partial \chi}{\partial r^{*}}\right)$ у уа que $d r=\gamma d r^{*}$ la acción $S$ se puede escribir

$$
\begin{array}{r}
S=\int c d t d r^{*} d \theta d \varphi \gamma r^{2} \sin \theta \\
\left(\frac{\left(\frac{1}{c^{2}}\left|\frac{\partial \chi}{\partial t}\right|^{2}-\left|\frac{\partial \chi}{\partial r^{*}}\right|^{2}\right)}{\gamma}-\frac{1}{r^{2}}\left|\frac{\partial \chi}{\partial \theta}\right|^{2}\right. \\
\left.-\frac{1}{r^{2} \sin ^{2} \theta}\left|\frac{\partial \chi}{\partial \varphi}\right|^{2}-m^{\prime 2}|\chi|^{2}\right)
\end{array}
$$

Haciendo el cambio de variable: $\psi=r \chi$, tenemos que

$$
\begin{gathered}
\frac{\partial \chi}{\partial t}=\frac{1}{r} \frac{\partial \psi}{\partial t} \\
\frac{\partial \chi}{\partial r^{*}}=\frac{\gamma}{r^{2}}\left(\frac{r}{\gamma} \frac{\partial \psi}{\partial r^{*}}-\psi\right) \\
\frac{\partial \chi}{\partial \theta}=\frac{1}{r} \frac{\partial \psi}{\partial \theta} \\
\frac{\partial \chi}{\partial \varphi}=\frac{1}{r} \frac{\partial \psi}{\partial \varphi}
\end{gathered}
$$

adicionalmente, ya que $\frac{d \ln r}{d r^{*}}=\frac{\gamma}{r}$, la ecuación (19) se puede escribir:

$$
\begin{array}{r}
S=\int c d t d r^{*} d \theta d \varphi\left(\operatorname { s i n } \theta \left(\frac{1}{c^{2}}\right.\right. \\
\left.\left|\frac{\partial \psi}{\partial t}\right|^{2}-\left|\frac{\partial \psi}{\partial r^{*}}-\frac{d \ln r}{d r^{*}} \psi\right|^{2}\right) \\
\left.-\frac{\gamma}{r^{2}}\left(\sin \theta\left|\frac{\partial \psi}{\partial \theta}\right|^{2}+\frac{1}{\sin \theta|\psi|^{2}-}\left|\frac{\partial \psi}{\partial \varphi}\right|^{2}\right)\right)
\end{array}
$$

que también se puede escribir

$$
\begin{aligned}
S=\int & c d t d r^{*} d \theta d \varphi\left(\operatorname { s i n } \theta \left(\frac{1}{c^{2}}\left|\frac{\partial \psi}{\partial t}\right|^{2}\right.\right. \\
& -\left|\frac{\partial \psi}{\partial r^{*}}\right|^{2}+2 \operatorname{Re}\left(\psi \frac{\partial \psi^{*}}{\partial r^{*}}\right) \frac{d \ln r}{d r^{*}} \\
& \left.-\left(\frac{d \ln r}{d r^{*}}\right)^{2}|\psi|^{2}\right)-\gamma m^{\prime 2} \sin \theta|\psi|^{2} \\
& \left.-\frac{\gamma}{r^{2}}\left(\sin \theta\left|\frac{\partial \psi}{\partial \theta}\right|^{2}+\frac{1}{\sin \theta}\left|\frac{\partial \psi}{\partial \varphi}\right|^{2}\right)\right)
\end{aligned}
$$

Utilizando integración por partes tenemos que

$$
\begin{array}{r}
\int d r^{*} \operatorname{Re}\left(\psi \frac{\partial \psi^{*}}{\partial r^{*}}\right)\left(\frac{d \ln r}{d r^{*}}\right)= \\
\left(\int d r^{*} \operatorname{Re}\left(\psi \frac{\partial \psi^{*}}{\partial r^{*}}\right)\right)\left(\frac{d \ln r}{d r^{*}}\right) \\
-\int d r^{*}\left(\int d r^{*} \operatorname{Re}\left(\psi \frac{\partial \psi^{*}}{\partial r^{*}}\right)\right) \frac{d}{d r^{*}}\left(\frac{d \ln r}{d r^{*}}\right)
\end{array}
$$

también es facil demostrar (usando asimismo integración por partes) que

$$
\int d r^{*} \operatorname{Re}\left(\psi \frac{\partial \psi^{*}}{\partial r^{*}}\right)=\frac{1}{2}|\psi|^{2}
$$

Introduciendo la última integral en (22) tenemos:

$$
\begin{array}{r}
\int d r^{*} \operatorname{Re}\left(\psi \frac{\partial \psi^{*}}{\partial r^{*}}\right)\left(\frac{d \ln r}{d r^{*}}\right)= \\
-\frac{1}{2} \int d r^{*}|\psi|^{2} \frac{d}{d r^{*}}\left(\frac{d \ln r}{d r^{*}}\right) \\
+\frac{1}{2}|\psi|^{2}\left(\frac{d \ln r}{d r^{*}}\right)
\end{array}
$$

donde el segundo término del lado derecho de la integral (24) está evaluado en el borde de la región en consideración y es por lo tanto igual a cero. Por lo tanto la acción 
(21) se puede escribir:

$$
\begin{aligned}
S=\int & c d t d r^{*} d \theta d \varphi\left(\operatorname { s i n } \theta \left(\frac{1}{c^{2}}\left|\frac{\partial \psi}{\partial t}\right|^{2}\right.\right. \\
& -\left|\frac{\partial \psi}{\partial r^{*}}\right|^{2}-|\psi|^{2} \frac{d}{d r^{*}}\left(\frac{d \ln r}{d r^{*}}\right) \\
& \left.-\left(\frac{d \ln r}{d r^{*}}\right)^{2}|\psi|^{2}\right)-\gamma m^{\prime 2} \sin \theta|\psi|^{2} \\
& \left.-\frac{\gamma}{r^{2}}\left(\sin \theta\left|\frac{\partial \psi}{\partial \theta}\right|^{2}+\frac{1}{\sin \theta}\left|\frac{\partial \psi}{\partial \varphi}\right|^{2}\right)\right)
\end{aligned}
$$

Busquemos una solución en variables separables para la ecuación (25) de la forma [11, 12]

$$
\psi=\sum_{l_{1}, m_{1}} R_{l_{1} m_{1}}\left(t, r^{*}\right) Y_{l_{1} m_{1}}(\theta, \varphi)
$$

donde $R_{l_{1} m_{1}}$ es una función que depende exclusivamente de $t \mathrm{y} r^{*}, Y_{l_{1} m_{1}}$ son los armónicos esféricos $\left(l_{1}=\right.$ $\left.0,1, \ldots \ldots . \infty \mathrm{y}-l_{1} \leq m_{1} \leq l_{1}\right)$. Ahora

$$
\begin{aligned}
\left|\frac{\partial \psi}{\partial t}\right|^{2} & =\left(\sum_{l_{1}, m_{1}} \frac{\partial R_{l_{1} m_{1}}^{*}\left(t, r^{*}\right)}{\partial t} Y_{l_{1} m_{1}}^{*}(\theta, \varphi)\right) \\
& \times\left(\sum_{l_{2}, m_{2}} \frac{\partial R_{l_{2} m_{2}}\left(t, r^{*}\right)}{\partial t} Y_{l_{2} m_{2}}(\theta, \varphi)\right)
\end{aligned}
$$

Usando la propiedad de normalización de los armónicos esféricos [11, 12]:

$$
\begin{array}{r}
\int_{0}^{\pi} \int_{0}^{2 \pi} \sin \theta d \theta d \phi Y_{l_{1} m_{1}}^{*}(\theta, \varphi) Y_{l_{2} m_{2}}(\theta, \varphi)= \\
\delta_{l_{1} l_{2}} \delta_{m_{1} m_{2}}
\end{array}
$$

donde $\delta_{l_{1} l_{2}}$ y $\delta_{m_{1} m_{2}}$ son los delta de Kronecker, tenemos que

$$
\begin{aligned}
\int_{0}^{\pi} \int_{0}^{2 \pi} \sin \theta d \theta d \phi\left|\frac{\partial \psi}{\partial t}\right|^{2}=\sum_{l_{1}, m_{1}} \sum_{l_{2}, m_{2}} \frac{\partial R_{l_{1} m_{1}}^{*}}{\partial t} \\
\times \frac{\partial R_{l_{2} m_{2}}}{\partial t} \delta_{l_{1} l_{2}} \delta_{m_{1} m_{2}}=\sum_{l, m}\left|\frac{\partial R_{l m}}{\partial t}\right|^{2}
\end{aligned}
$$

donde $l=l_{1}=l_{2}, m=m_{1}=m_{2}$. Similarmente,

$$
\int_{0}^{\pi} \int_{0}^{2 \pi} \sin \theta d \theta d \phi\left|\frac{\partial \psi}{\partial r^{*}}\right|^{2}=\sum_{l, m}\left|\frac{\partial R_{l m}}{\partial r^{*}}\right|^{2}
$$

y,

$$
\int_{0}^{\pi} \int_{0}^{2 \pi} \sin \theta d \theta d \phi|\psi|^{2}=\sum_{l, m}\left|R_{l m}\right|^{2} .
$$

adicionalmente,

$$
\begin{array}{r}
\frac{\partial \psi}{\partial \theta}=\sum_{l_{1}, m_{1}} R_{l_{1} m_{1}}\left(t, r^{*}\right) \\
\times \frac{d}{d \theta}\left(P_{l_{1}, m_{1}}(\cos \theta)\right) \frac{1}{\sqrt{2 \pi}} e^{i m_{1} \varphi},
\end{array}
$$

$$
\begin{array}{r}
\frac{\partial \psi}{\partial \varphi}=\sum_{l_{1}, m_{1}} R_{l_{1} m_{1}}\left(t, r^{*}\right) \\
\times\left(P_{l_{1}, m_{1}}(\cos \theta)\right) \frac{1}{\sqrt{2 \pi}} i m_{1} e^{i m_{1} \varphi}
\end{array}
$$

donde $\operatorname{los} P_{l_{1}, m_{1}}(\cos \theta)$ son los polinomios asociados de Legendre normalizados. Usando

$$
\int_{0}^{2 \pi} e^{i\left(m_{2}-m_{1}\right) \varphi} d \varphi=2 \pi \delta_{m_{1} m_{2}}
$$

resulta que

$$
\begin{array}{r}
\int_{0}^{\pi} \int_{0}^{2 \pi} \sin \theta d \theta d \phi\left|\frac{\partial \psi}{\partial \theta}\right|^{2}= \\
\sum_{l_{1}, l_{2}, m_{1}} R_{l_{1} m_{1}}^{*}\left(t, r^{*}\right) R_{l_{2} m_{1}}\left(t, r^{*}\right) \int_{0}^{\pi} \sin \theta \\
\frac{d}{d \theta}\left(P_{l_{1}, m_{1}}(\cos \theta)\right) \frac{d}{d \theta}\left(P_{l_{2}, m_{1}}(\cos \theta)\right) d \theta
\end{array}
$$

Utilizando la fórmula de integración por partes tenemos:

$$
\begin{array}{r}
\int_{0}^{\pi} \sin \theta \frac{d}{d \theta}\left(P_{l_{1}, m_{1}}(\cos \theta)\right) \\
\times \frac{d}{d \theta}\left(P_{l_{2}, m_{1}}(\cos \theta)\right) d \theta=-\int_{0}^{\pi} P_{l_{1}, m_{1}}(\cos \theta) \\
\times \frac{d}{d \theta}\left(\sin \theta \frac{d}{d \theta}\left(P_{l_{2}, m_{1}}(\cos \theta)\right)\right) d \theta
\end{array}
$$

Introduciendo la ecuación (36) en la expresión (35) y empleando la ecuación diferencial de los polinomios asociados de Legendre [11]:

$$
\begin{gathered}
\frac{d}{d \theta}\left(\sin \theta \frac{d}{d \theta}\left(P_{l, m}(\cos \theta)\right)\right)= \\
\left(\frac{m^{2}}{\sin \theta}-l(l+1) \sin \theta\right) P_{l, m}(\cos \theta)
\end{gathered}
$$

tenemos que:

$$
\begin{array}{r}
\int_{0}^{\pi} \int_{0}^{2 \pi} \sin \theta d \theta d \phi\left|\frac{\partial \psi}{\partial \theta}\right|^{2}= \\
-\sum_{l_{1}, l_{2}, m_{1}} R_{l_{1} m_{1}}^{*}\left(t, r^{*}\right) R_{l_{2} m_{1}}\left(t, r^{*}\right) \\
\times \int_{0}^{\pi} d \theta P_{l_{1}, m_{1}}(\cos \theta) P_{l_{2} m_{1}}(\cos \theta) \\
\times\left(\frac{m_{1}^{2}}{\sin \theta}-l_{2}\left(l_{2}+1\right) \sin \theta\right)
\end{array}
$$

Similarmente

$$
\begin{array}{r}
\int_{0}^{\pi} \int_{0}^{2 \pi} \frac{1}{\sin \theta} d \theta d \phi\left|\frac{\partial \psi}{\partial \varphi}\right|^{2}= \\
\sum_{l_{1}, l_{2}, m_{1}} R_{l_{1} m_{1}}^{*}\left(t, r^{*}\right) R_{l_{2} m_{1}}\left(t, r^{*}\right) \\
\times \int_{0}^{\pi} d \theta\left(\frac{m_{1}^{2}}{\sin \theta}\right) P_{l_{1}, m_{1}}(\cos \theta) P_{l_{2} m_{1}}(\cos \theta)
\end{array}
$$


Los polinomios asociados de Legendre normalizados satisfacen la relación [11]

$\int_{0}^{\pi} \sin \theta P_{l_{1}, m_{1}}(\cos \theta) P_{l_{2}, m_{1}}(\cos \theta) d \theta=\delta_{l_{1} l_{2}}$

de manera que si sumamos las ecuaciones 38 y 39 ,utilizando la expresión 40) tenemos que:

$$
\begin{array}{r}
\int_{0}^{\pi} \int_{0}^{2 \pi} d \theta d \phi\left(\sin \theta\left|\frac{\partial \psi}{\partial \theta}\right|^{2}+\frac{1}{\sin \theta}\left|\frac{\partial \psi}{\partial \varphi}\right|^{2}\right) \\
=\sum_{l, m}\left|R_{l, m}\right|^{2} l(l+1)
\end{array}
$$

donde $l_{1}=l$ y $m_{1}=m$. Introduciendo las integrales 29,30, 31, 41) en la ecuación (25) tenemos:

$$
\begin{array}{r}
S=\int c d t d r^{*} \sum_{l, m}\left(\frac{1}{c^{2}}\left|\frac{\partial R_{l, m}}{\partial t}\right|^{2}-\left|\frac{\partial R_{l, m}}{\partial r^{*}}\right|^{2}\right. \\
-\left|R_{l, m}\right|^{2}\left(\frac{d}{d r^{*}}\left(\frac{d \ln r}{d r^{*}}\right)+\left(\frac{d \ln r}{d r^{*}}\right)^{2}\right) \\
\left.-\gamma\left(\frac{1}{r^{2}} l(l+1)+m^{\prime 2}\right)\left|R_{l, m}\right|^{2}\right)
\end{array}
$$

Por otro lado

$$
\left(\frac{d \ln r}{d r^{*}}\right)^{2}=\frac{1}{r^{2}}\left(1-\frac{2 G M}{r c^{2}}\right)^{2}
$$

$\mathrm{y}$

$$
\begin{array}{r}
\frac{d}{d r^{*}}\left(\frac{d \ln r}{d r^{*}}\right)=\frac{1}{r^{2}} \\
\times\left(\frac{4 G M}{r c^{2}}-1\right)\left(1-\frac{2 G M}{r c^{2}}\right) .
\end{array}
$$

Con estas derivadas, la ecuación (42) se puede escribir:

$$
\begin{array}{r}
S=\int c d t d r^{*} \sum_{l, m}\left(\frac{1}{c^{2}}\left|\frac{\partial R_{l, m}}{\partial t}\right|^{2}-\left|\frac{\partial R_{l, m}}{\partial r^{*}}\right|^{2}\right. \\
\left.-V_{e f}^{l}\left(r^{*}\right)\left|R_{l, m}\right|^{2}\right)
\end{array}
$$

donde el potencial efectivo $V_{e f}^{l}\left(r^{*}\right)$ está definido por:

$$
\begin{array}{r}
V_{e f}^{l}\left(r^{*}\right)=\left(1-\frac{2 G M}{r c^{2}}\right) \\
\times\left(\frac{2 G M}{r^{3} c^{2}}+\frac{l(l+1)}{r^{2}}+m^{\prime 2}\right)
\end{array}
$$

Fácilmente podemos ver que si $r=\frac{2 G M}{c^{2}}$ entonces $V_{e f}^{l}\left(r^{*}\right)=0$.

\section{Ecuación de onda}

A partir de la acción dada por la expresión (45) podemos escribir la ecuación de Euler-Lagrange [13] correspondiente a la parte radial del campo escalar complejo masivo $\chi$

$$
\frac{\partial L}{\partial R_{l, m}^{*}}-\partial_{\mu}\left(\frac{\partial L}{\partial\left(\partial_{\mu} R_{l, m}^{*}\right)}\right)=0
$$

donde $L$ está dado por

$$
\begin{array}{r}
L=\frac{1}{c^{2}}\left|\frac{\partial R_{l, m}}{\partial t}\right|^{2}-\left|\frac{\partial R_{l, m}}{\partial r^{*}}\right|^{2} \\
-V_{e f}^{l}\left(r^{*}\right)\left|R_{l, m}\right|^{2} .
\end{array}
$$

En forma desarrollada la ecuación (47) es:

$$
\begin{aligned}
\frac{\partial L}{\partial R_{l, m}^{*}}- & \frac{1}{c} \frac{\partial}{\partial t}\left(\frac{\partial L}{\partial\left(\frac{1}{c} \frac{\partial R_{l, m}^{*}}{\partial t}\right)}\right)- \\
& -\frac{\partial}{\partial r}\left(\frac{\partial L}{\partial\left(\frac{\partial R_{l, m}^{*}}{\partial r}\right)}\right)=0
\end{aligned}
$$

que se reduce empleando la ecuación (14) a:

$$
\frac{1}{c^{2}} \frac{\partial^{2} R_{l, m}}{\partial t^{2}}=\frac{\partial^{2} R_{l, m}}{\partial r^{* 2}}-V_{e f}^{l}\left(r^{*}\right) R_{l, m},
$$

que es la ecuación de onda correspondiente a la parte radial del campo escalar complejo masivo $\chi$.

\section{Potencial efectivo}

Para hallar los puntos críticos en el potencial efectivo tenemos que calcular la derivada de la función $V_{e f}^{l}$ con respecto a $r$, el resultado es la ecuación de tercer grado:

$$
r^{3}-\frac{2 b}{a m^{\prime 2}} r^{2}+\frac{3(b-1)}{m^{\prime 2}} r+\frac{4 a}{m^{\prime 2}}=0
$$

donde $a=\frac{2 G M}{c^{2}}$ y $b=l(l+1)$. Notemos que $a, b>0$. Las raices de esta ecuación son [14, 15]

$$
r_{1}=S+T-\frac{1}{3} a_{1}
$$

$$
\begin{aligned}
& r_{2}=-\frac{1}{2}(S+T)-\frac{1}{3} a_{1}+\frac{1}{2} i \sqrt{3}(S-T) \\
& r_{3}=-\frac{1}{2}(S+T)-\frac{1}{3} a_{1}-\frac{1}{2} i \sqrt{3}(S-T)
\end{aligned}
$$

donde $S \equiv\left(R+D^{\frac{1}{2}}\right)^{\frac{1}{3}} ; T \equiv\left(R-D^{\frac{1}{2}}\right)^{\frac{1}{3}} ; D \equiv$ $Q^{3}+R^{2}$ y $a_{1}=-\frac{2 b}{a m^{\prime 2}}$, con

$$
Q=\frac{9 a^{2} m^{\prime 2}(b-1)-4 b^{2}}{9 a^{2} m^{\prime 4}}
$$

$\mathrm{y}$

$$
R=\frac{-54 b(b-1) a^{2} m^{\prime 2}-108 a^{4} m^{4}+16 b^{3}}{54 a^{3} m^{\prime 6}} .
$$


Introduciendo los valores de $Q$ y $R$ en $D \equiv Q^{3}+R^{2}$ obtenemos:

$$
\begin{aligned}
D & =\frac{1}{2916 a^{2} m^{\prime 8}}\left(2916 a^{2} m^{\prime 2}(b-1)(b+1)^{2}\right. \\
& \left.-b^{2}\left(972 b^{2}+1512 b+972\right)+11664 a^{4} m^{\prime 4}\right)
\end{aligned}
$$

Dependiendo de los valores de $D$, las raíces de la ecuación de tercer grado 51 pueden ser reales o complejas. Asi:

i) Si $D>0 \quad r_{1}$ es real y $r_{2}, r_{3}$ son complejas conjugadas.

ii) Si $D=0 \quad r_{1}, r_{2}$ y $r_{3}$ son reales y adicionalmente $r_{2}=r_{3}$.

iii) Si $D<0 \quad r_{1}, r_{2}$ y $r_{3}$ son reales y distintas.

Por supuesto a nosotros solo nos interesan las raíces reales. Por cierto, en el caso $D<0$ las raíces son:

$$
\begin{gathered}
r_{1}=2(-Q)^{\frac{1}{2}} \cos \left(\frac{\theta}{3}\right)-\frac{1}{3} a_{1} \\
r_{2}=2(-Q)^{\frac{1}{2}} \cos \left(\frac{\theta}{3}+\frac{2 \pi}{3}\right)-\frac{1}{3} a_{1} \\
r_{3}=2(-Q)^{\frac{1}{2}} \cos \left(\frac{\theta}{3}+\frac{4 \pi}{3}\right)-\frac{1}{3} a_{1}
\end{gathered}
$$

donde $\cos \theta=-\frac{R}{\left(-Q^{3}\right)^{\frac{1}{2}}}$.

Por cierto, en el caso particular en el que $l=0(b=0)$ la ecuación 57 se reduce a

$$
D=\frac{1}{m^{\prime 6}}\left(2 a m^{\prime}-1\right)\left(2 a m^{\prime}+1\right)
$$

\section{Campo escalar complejo no masivo}

En el caso en el que el campo escalar complejo no tiene masa $\left(m^{\prime}=\frac{m_{0} c}{\hbar}=0\right)$ el máximo del potencial efectivo $V_{e f}^{l}\left(r^{*}\right)$ (ver ecuación 46) ocurre para

$$
\begin{gathered}
r_{\max }=\frac{3 G M}{c^{2}}\left(\frac{l(l+1)-1}{2 l(l+1)}\right. \\
\left.+\frac{1}{2}\left(1+\frac{\left(14 l^{2}+14 l+9\right)}{9 l^{2}(l+1)^{2}}\right)^{\frac{1}{2}}\right)
\end{gathered}
$$

que en el límite cuando $l \rightarrow \infty$ se reduce a

$$
\lim _{l \rightarrow \infty}\left(r_{\max }\right)=\frac{3 G M}{c^{2}}
$$

Para $r>>\frac{3 G M}{c^{2}}$ el potencial efectivo es repulsivo. Sin embargo, a medida que nos aproximamos al horizonte en $r=\frac{2 G M}{c^{2}}$ la atracción gravitacional comienza a dominar y el potencial se vuelve atractivo empujando el paquete de ondas hacia el horizonte. En el límite cuando $l \rightarrow 0$ el máximo del potencial efectivo es

$$
\begin{array}{r}
r_{\max }=\frac{3 G M}{2 c^{2}}\left(1+\lim _{l \rightarrow 0}\left(-\frac{1}{l(l+1)}+\right.\right. \\
\left.\left.+\left(1+\frac{\left(14 l^{2}+14 l+9\right)}{9 l^{2}(l+1)^{2}}\right)^{\frac{1}{2}}\right)\right) \\
=\frac{8 G M}{3 c^{2}}<\frac{3 G M}{c^{2}}
\end{array}
$$

El potencial efectivo correspondiente es:

$$
V_{e f}^{l=0}\left(r_{\max }\right)=\frac{1}{2}\left(\frac{3}{8}\right)^{3} \frac{c^{4}}{G^{2} M^{2}}
$$

Para valores grandes de $l$ también podemos escribir aproximadamente

$$
V_{e f}^{l}\left(r_{\max }\right) \approx \frac{1}{27} \frac{c^{4} l^{2}}{G^{2} M^{2}}
$$

A continuación escribimos los valores de $r_{\max }$ y el potencial efectivo para algunos valores de $l$

$$
\begin{gathered}
r_{\max }(l=1)=\frac{3 G M}{4 c^{2}}\left(1+\frac{1}{3} \sqrt{73}\right)<\frac{3 G M}{c^{2}} \\
V_{e f}^{l=1}\left(r_{\max }\right)=0,09926 \frac{c^{4}}{G^{2} M^{2}} \\
r_{\max }(l=2)=\frac{3 G M}{c^{2}}\left(\frac{15+\sqrt{417}}{36}\right)<\frac{3 G M}{c^{2}} \\
V_{e f}^{l=2}\left(r_{\max }\right)=0,24712 \frac{c^{4}}{G^{2} M^{2}}
\end{gathered}
$$

En la figura 1 representamos el potencial efectivo $V_{e f}^{l}$ en términos de la distancia radial $r$ y $l$ para $m^{\prime}=0$.

\section{Campo escalar complejo masivo}

Las raíces de la ecuación (51) para un campo escalar complejo masivo $\left(m^{\prime} \neq 0\right)$ en el caso $l=0(b=0)$ son:

i) Si $D>0$ o $m^{\prime}>\frac{1}{2 a}=\frac{c^{2}}{4 G M}$ (ver ecuación 61) la única raiz real es:

$$
\begin{gathered}
r_{1}=\left(\frac{1}{m^{\prime 3}}\left(\left(\frac{4 G M m^{\prime}}{c^{2}}\right)^{2}-1\right)^{\frac{1}{2}}-\frac{4 G M}{c^{2} m^{\prime 2}}\right)^{\frac{1}{3}} \\
+\left(-\frac{1}{m^{\prime 3}}\left(\left(\frac{4 G M m^{\prime}}{c^{2}}\right)^{2}-1\right)^{\frac{1}{2}}-\frac{4 G M}{c^{2} m^{\prime 2}}\right)^{\frac{1}{3}}
\end{gathered}
$$

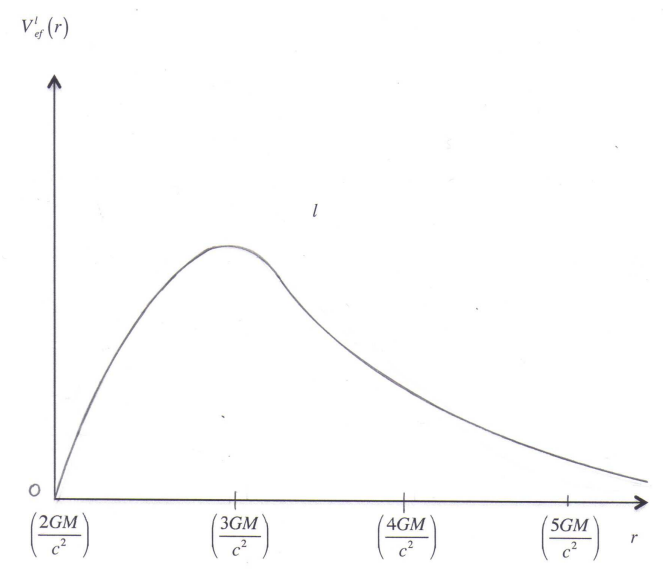

Figura 1: Gráfica del potencial efectivo $V_{\text {ef }}^{1}$ en términos de la distancia radial $\mathbf{r}$ y $\mathbf{l}$ para $\mathrm{m}^{\prime}=0$. 
pero fácilmente se puede chequear que $r_{1}<0$ por lo que tenemos que desechar dicha solución ( $r$ es definido positivo).

ii) Si $D=0$ o $m^{\prime}=\frac{1}{2 a}=\frac{c^{2}}{4 G M}$ solo hay una raiz real

$$
r_{2}=r_{3}=r^{\prime}=\left(\frac{4 G M}{c^{2} m^{\prime 2}}\right)^{\frac{1}{3}}=\frac{1}{m^{\prime}}=\frac{4 G M}{c^{2}} .
$$

El potencial efectivo correspondiente es:

$$
V_{e f}^{l=0}=\frac{3}{4} m^{\prime 2}
$$

Sin embargo, ya que para $r^{\prime}$ se cumple que $\frac{d^{2} V_{e f}^{l=0}}{d r^{2}}=0$, dicha raiz es un punto de inflexión de la curva de potencial efectivo.Por otro lado, $\lim _{r \rightarrow \infty} V_{e f}^{l=0}=m^{\prime 2}$ es una asíntota horizontal de la función de potencial efectivo.

En la figura 2 se representa dicho potencial efectivo en términos de la distancia radial $r$ para $l=0$.

iii) Si $D<0$ o $m^{\prime}<\frac{1}{2 a}=\frac{c^{2}}{4 G M}$ la raiz es

$$
r=\left(\frac{2}{m^{\prime}}\right) \cos \left(\frac{\theta}{3}\right)=\frac{2 \hbar}{m_{0} c} \cos \left(\frac{\theta}{3}\right),
$$

donde $\cos \left(\frac{\theta}{3}\right)>\frac{G M m^{\prime}}{c^{2}}$. El potencial efectivo es en este caso:

$$
\begin{aligned}
V_{e f}^{l=0} \equiv V_{e f}^{*}=m^{\prime 2} & +\left(\frac{2 G m}{c^{2}}\right)\left(\frac{m^{\prime}}{2 \cos \left(\frac{\theta}{3}\right)}\right) \times \\
& \times\left(\left(\frac{m^{\prime}}{2 \cos \left(\frac{\theta}{3}\right)}\right)^{2}-m^{\prime 2}\right. \\
& \left.-\left(\frac{2 G m}{c^{2}}\right)\left(\frac{m^{\prime}}{2 \cos \left(\frac{\theta}{3}\right)}\right)^{3}\right)
\end{aligned}
$$

donde

$$
\cos (\theta)=\frac{4 G M m_{0}}{c \hbar}
$$

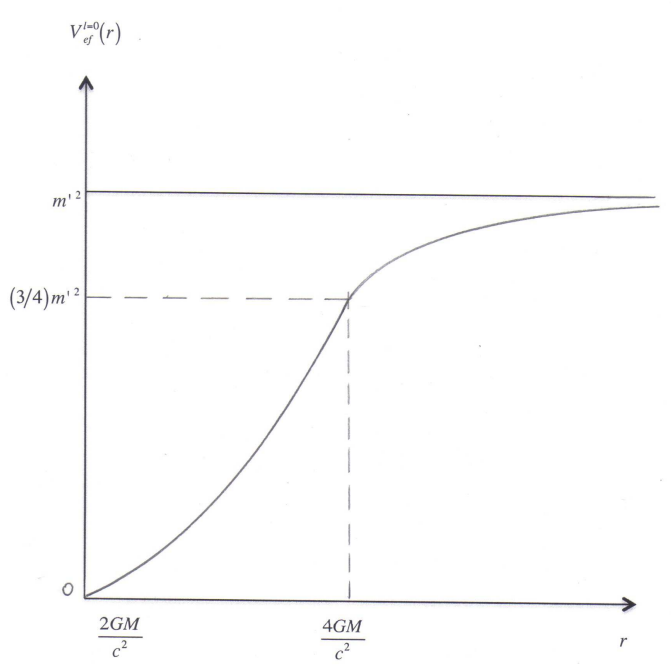

Figura 2: Gráfica del potencial efectivo $V_{\text {ef }}^{1}$ en términos de la distancia radial $\mathrm{r}$ para $\mathrm{m}^{\prime} \neq 0, \mathrm{D}=0$ y $\mathrm{l} \stackrel{\text { ef }}{=} 0$.

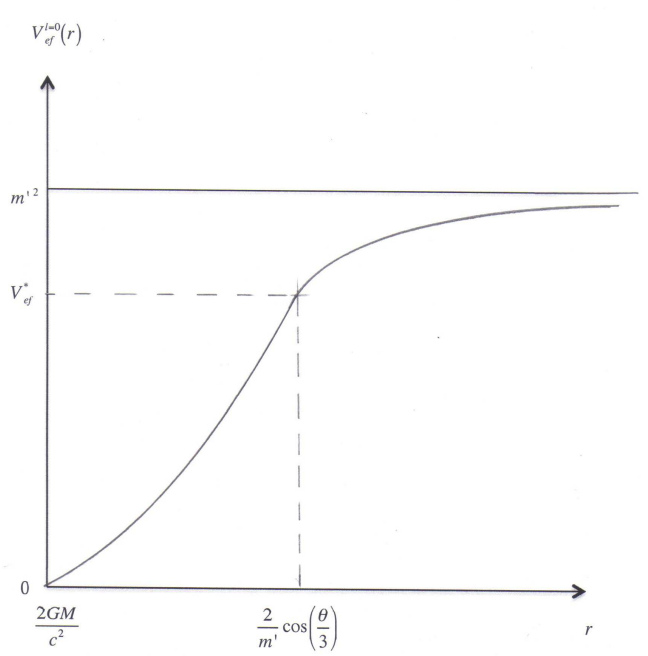

Figura 3: Gráfica del potencial efectivo $\mathrm{V}_{\text {ef }}^{1}$ en términos de la distancia radial $\mathrm{r}$ para $\mathrm{m}^{\prime} \neq 0, \mathrm{D}<0$ y $\mathrm{l} \stackrel{\text { ef }}{=} 0$.

La gráfica del potencial en términos de $r$ para $l=0$ se representa en la figura 3 .

\section{Solución de la ecuación de onda}

Retornando a la ecuación de onda (50), para un modo de frecuencia $\omega$ escribiremos

$$
R_{l, m}\left(t, r^{*}\right)=e^{-i \omega t} R_{l, m}\left(r^{*}\right)
$$

que al ser introducida en la ecuación de onda mencionada se transforma en la ecuación:

$$
\frac{d^{2} R_{l, m}}{d r^{* 2}}-V_{e f}^{l}\left(r^{*}\right) R_{l, m}\left(r^{*}\right)=-\frac{\omega^{2}}{c^{2}} R_{l, m}\left(r^{*}\right)
$$

con $-l \leq m \leq l ; l=0,1, \ldots \ldots . \infty$.

Para grandes valores de $r, V_{e f}^{l}\left(r^{*}\right)=m^{\prime 2}$, por lo que podemos escribir

$$
\frac{d^{2} R_{l, m}}{d r^{* 2}}+\left(\frac{\omega^{2}}{c^{2}}-m^{\prime 2}\right) R_{l, m}\left(r^{*}\right)=0 .
$$

Si $\frac{\omega}{c} \geq m^{\prime}$ o $E \geq m_{0} c^{2}$ la solución de la ecuación (79) es

$$
R_{l, m}\left(r^{*}\right)=A_{ \pm} e^{ \pm i\left(\frac{\omega^{2}}{c^{2}}-m^{\prime 2}\right)^{\frac{1}{2}} r^{*}}
$$

donde los $A_{ \pm}$son constantes de normalización. Por lo tanto tenemos que

$$
R_{l, m}\left(t, r^{*}\right)=A_{ \pm} e^{-i k c\left(t \mp\left(1-\frac{m^{\prime 2}}{k^{2}}\right)^{\frac{1}{2}} \frac{r^{*}}{c}\right)}
$$

donde $k=\frac{\omega}{c}$ representa la magnitud del vector de onda. Los modos de oscilación en esta región tienen la forma de ondas planas que se propagan con velocidad

$$
\frac{d r^{*}}{d t}=c\left(1-\frac{m^{\prime 2}}{k^{2}}\right)^{-\frac{1}{2}}
$$


Por otro lado, si $\frac{\omega}{c} \leq m^{\prime}$ o $E<m_{0} c^{2}$ la solución de la ecuación (79) es

$$
R_{l, m}\left(r^{*}\right)=B e^{-k r\left(\left(\frac{m^{\prime}}{k}\right)^{2}-1\right)^{\frac{1}{2}}}
$$

donde $B$ es una constante de normalización.Entonces

$$
R_{l, m}\left(t, r^{*}\right)=B e^{-i k c t} e^{-k r\left(\left(\frac{m^{\prime}}{k}\right)^{2}-1\right)^{\frac{1}{2}}}
$$

A partir de las ecuaciones (81) y 84) podemos calcular la amplitud de probabilidad de que la partícula penetre la barrera de potencial.

Ahora bien, en el caso en el que $m^{\prime}=0$, y a medida que nos aproximamos al horizonte de sucesos del agujero negro $\left(r \rightarrow \frac{2 G M}{c^{2}}\right)$ la coordenada $r^{*}$ se hace infinitamente grande y negativa. En este caso el potencial es despreciable $\left(V_{e f}^{l}\left(r^{*}\right) \rightarrow 0\right)$, y entonces el campo se comporta como un campo libre de Klein-Gordon sin masa. Entonces la ecuación (78) se escribe:

$$
\frac{d^{2} R_{l, m}}{d r^{* 2}}=-\frac{\omega^{2} c^{2}}{R_{l, m}}\left(r^{*}\right)
$$

cuya solución es

$$
R_{l, m}\left(r^{*}\right)=A_{ \pm} e^{ \pm i k r^{*}}
$$

de manera que

$$
R_{l, m}\left(t, r^{*}\right)=A_{ \pm} e^{-i k c\left(t \mp \frac{r^{*}}{c}\right)}
$$

donde $A_{ \pm}$es una constante de normalización.En esta región los modos de oscilación son ondas planas que se propagan a la velocidad de la luz:

$$
\frac{d r^{*}}{d t}= \pm c
$$

\section{Escapando de la barrera de potencial}

En la sección: Campo escalar complejo no masivo, calculamos la altura de la barrera de potencial para $m^{\prime}=0$. Por ejemplo para $l=0$, dicha altura era

$$
V_{e f}^{l=0}\left(r_{\max }\right)=\frac{1}{2}\left(\frac{3}{8}\right)^{3} \frac{c^{4}}{G^{2} M^{2}}
$$

Entonces, una onda del tipo $s$ ( $s$-wave quantum) escapará de la mencionada barrera si

$$
\frac{\omega}{c}>\left(\frac{1}{2}\left(\frac{3}{8}\right)^{3}\right)^{\frac{1}{2}} \frac{c^{2}}{G M}=0,1623 \frac{c^{2}}{G M}
$$

o si la energía $E$ satisface:

$$
E=\omega \hbar>0,1623 \frac{c^{3} \hbar}{G M}
$$

Similarmente para $l=1$ (una onda del tipo $p$ ) la condición para atravesar la barrera es:

$$
E=\omega \hbar>0,315 \frac{c^{3} \hbar}{G M}
$$

Para $l=2$ la condición es

$$
E=\omega \hbar>0,4971 \frac{c^{3} \hbar}{G M}
$$

Vemos entonces que a medida que el valor de $l$ se incrementa, la energía que se necesita para atravesar la barrera de potencial aumenta. Por cierto, una onda será capaz de penetrar la barrera desde el exterior y caer en el horizonte de sucesos (para $l=0,1$ y 2 , respectivamente) si se cumplen las mismas condiciones dadas en las ecuaciones (90), 91) y 92).

Para grandes valores de $l$, el umbral de energía para pasar sobre la barrera de potencial es (ver ecuación 66) $E \geq \frac{c^{3} l \hbar}{\sqrt{27} G M}$

Para $m^{\prime} \neq 0$ en el caso $D=0, l=0$ teníamos que

$$
m^{\prime}=\frac{c^{2}}{4 G M}=\frac{m_{0} c}{\hbar}
$$

de manera que

$$
m_{0}=\frac{\hbar c}{4 G M}
$$

La altura de la barrera era $V_{e f}^{l=0}(\max )=m^{\prime 2}$, de manera que una onda del tipo $s$ escapará del horizonte de eventos si $\frac{\omega}{c}>m^{\prime}$ o equivalentemente si

$$
E>m_{0} c^{2}=\frac{\hbar c^{3}}{4 G M}
$$

Adicionalmente, una onda de dicho tipo que satisfaga la condición dada por la ecuación (94) podrá penetrar la barrera desde el exterior y caer en el horizonte de eventos

\section{Conclusiones}

En este artículo hemos analizado el comportamiento de un campo escalar complejo masivo de Klein-Gordon inmerso en una background de Schwarzschild usando coordenadas de tortuga (Tortoise coordinates). Con la introducción de dichas coordenadas podemos cubrir la región $r>\frac{2 G M}{c^{2}}$ sin preocuparnos de lo que suceda en la región por debajo del radio de Schwarzschild. Usando dichas coordenadas obtuvimos una expresión para la acción en términos de un potencial efectivo $V_{e f}^{l}\left(r^{*}\right)$ y una función de onda radial $R_{l, m}\left(t, r^{*}\right)$. A partir de las ecuaciones de Euler Lagrange dedujimos la ecuación de onda para $R_{l, m}\left(t, r^{*}\right)$ y el potencial efectivo. Posteriormente estudiamos el comportamiento del potencial en función de la distancia radial $r$ tanto para $m^{\prime}=0$ (campo escalar complejo sin masa) como para $m^{\prime} \neq 0$ (campo escalar complejo masivo) y diferentes valores del número cuántico $l$. En las figuras 12 y 3 se representa el 
potencial efectivo para $m^{\prime}=0$ para un valor de $l$ cualquiera y para $m^{\prime} \neq 0$ en los casos $D=0, l=0$ (ver ecuación 57 para la definición de $D$ ) y $D<0, l=0$, respectivamente.

Para un modo de frecuencia $\omega$ escribimos la ecuación de onda correspondiente y calculamos algunas soluciones de dicha ecuación para $\frac{\omega}{c} \geq m^{\prime}$ y $\frac{\omega}{c}<m^{\prime}$. También escribimos la solución correspondiente a $m^{\prime}=$ 0.Finalmente, evaluamos la energía necesaria para atravesar la barrera de potencial para algunos valores del número cuántico $l$.

Para $m^{\prime}=0$, por ejemplo, la energía que se necesita para atravesar la barrera de potencial aumenta a medida que el valor de $l$ crece.Para $r>>\frac{3 G M}{c^{2}}$ el potencial efectivo es repulsivo. Sin embargo, a medida que nos aproximamos al horizonte en $r=\frac{2 G M}{c^{2}}$ la atracción gravitacional comienza a dominar y el potencial se vuelve atractivo empujando el paquete de ondas hacia el horizonte.

Para $m^{\prime} \neq 0$ en el caso $D=0, l=0$ el potencial efectivo tiene una asíntota horizontal $V_{e f}^{l=0}(\max )=m^{\prime 2}$ de manera que una onda del tipo $s$ escapará del horizonte de eventos si $E>\frac{\hbar c^{3}}{4 G M}$. A su vez, una onda será capaz de penetrar la barrera de potencial desde el exterior y caer en el horizonte de eventos si se cumplen las mismas condiciones de energía que se necesitan para escapar del mencionado horizonte.

\section{Referencias bibliográficas}

[1] Ludvigsen, M. 1999. "General Relativity A Geometric Approach", Cambridge University Press, Cambridge, $U K$.

[2] Kenyon, I. 1996. "General Relativity", Oxford University Press, Oxford, UK.

[3] Marín, C. 2007. "La Expansión del Universo", Una Introducción a Cosmología, Relatividad General y Física de Partículas, USFQ, Quito-Ecuador.

[4] Ohanian, H. 1976. "Gravitation and Spacetime", W.W. Norton \& Company, Inc.

[5] Misner, C.; Thorne, K.; Wheeler, J. 1973. "Gravitation", W. H. Freeman \& Company, $N Y$.

[6] Cheng, T. 2005. "Relativity, Gravitation and Cosmology, A Basic Introduction", Oxford University Press, Oxford ,UK.

[7] Ryder, L. 1991. "Quantum Field Theory", Cambridge University Press, Cambridge, UK.

[8] Kaku, M. 1993. "Quantum Field Theory,A Modern Introduction", Oxford University Press, Oxford, UK.

[9] Halzen, F.; Martin, A. 1984. "Quarks and Leptons", John Wiley \& Sons.

[10] Susskind, L.; Lindesay, J. 2005. "An Introduction to Black Holes, Information and the String Theory Revolution, The Holographic Universe", World Scientific Publishing.
[11] Arfken, G.; Weber, H. 2005. "Mathematical Methods for Physicists", Sixth Edition, Academic Press.

[12] Riley, K.; Hobson, M.; Bence, S. 2006. "Mathematical Methods for Physics and Engineering", Third Edition, Cambridge University Press, Cambridge, UK.

[13] Hobson, M.; Efstathiou, G.; Lasenby, A. 2006. "General Relativity, An Introduction for Physicists", Cambridge University Press, Cambridge, UK.

[14] Spiegel, M.; Rapun, L. 1988. "Fórmulas y Tablas de Matemática Aplicada", Mc Graw Hill.

[15] Beyer, W. 1991. "Standard Mathematical Tables and Formulae", 29th Edition, CRC Press. 